\title{
Laparoscopic Approach through Incisional or Umbilical Hernia Sac
}

\author{
Mihaela Leșe* \\ Emergency County Hospital Baia Mare, Romania Mihaela Leşe, MD General Surgery \\ Department Emergency County Hospital Baia MareMaramureș, Romania \\ *Corresponding author
}

A B S T R A C T

Keywords

Incisional hernia,

Gigantic hernia,

Laparoscopic

approach, Gastric

polyp, Acute

cholecystitis,

Emergency surgery

Article Info

Accepted:

22 January 2019

Available Online:

10 February 2019
Laparoscopic surgery is constantly trying to progress and to surpass its contraindications. A previously operated abdomen or peritoneal adhesions represent nowadays only relative contraindications for a laparoscopic approach in abdominal visceral surgeries not primarily intended to correct parietal defects or to perform adhesiolysis. We present the laparoscopic approach through the incisional herni aand the giant umbilical hernia sac in 2 patients in need of emergency surgery for pathology. We support this laparoscopic approach in the following cases: the parietal defect cannot be repaired during the same intervention, it is of utmost importance to avoid the classical approach because of the increased risk for parietal and general complications, and the team has enough experience in the laparoscopic technique and the equipment functions flawlessly.

\section{Introduction}

Because of its numerous advantages for the patients, the laparoscopic approach represents in most cases the first choice in elective surgery, but also in emergency surgery. Among the most important short-term advantages of this type of technique are pain reliefs, decreasing the risk of postoperative ileus, lowering the rate of wound infection, quick recovery, as well as shortened length of stay in hospital. For the long-term, the greatest advantage is the reduced risk for postoperative incisional hernias which usually occur after conventional surgery and represents a big challenge for every surgeon, especially because of the high recurrence rate (1).

The question is, if the laparoscopic approach is so beneficial why it is not used in all the patients. There are mainly two reasons: the surgical team ability in performing laparoscopic surgeries and the patient complexity (2). Of the possible complex situations in a patient, previous open surgery, the presence of adhesions and abdominal 
parietal defects are some of the most important risk factors for conversion or relative contraindications for laparoscopic surgery $(3,4)$.

This case report will present the emergency laparoscopic surgery performed for other conditionsin two patients with major abdominal wall defects. They were hospitalised for a hemorrhagic and an infectious complication of their underlying pathology, this being different than the abdominal wall defect.

\section{Case 1}

A 77-year-old female patient is admitted through the emergency department for upper digestive tract bleeding symptoms: melena and fatigue. She also presents a high-risk $3^{\text {rd }}$ grade arterial hypertension and an uncomplicated bulky incisional hernia after an open cholecystectomy performed 20 years ago. The complete blood count showed hemoglobin of $9.1 \mathrm{~g} / \mathrm{dl}$, hematocrit of $26.5 \%$ and platelet count of 76,000/ $\mu 1$. The abdominal ultra Sound depicted cirrhosis of the liver and splénomégalie, without ascetic fluid. An emergency upper gastrointestinal endoscopie was performed, revealing blood in the stomach, and an approximately $3.5 \mathrm{~cm}$ polyp, with a large base, was identified in the pyloric antrum. $10 \mathrm{ml}$ Adrenaline 1:10,000 was injected, but its large implantation base prevented the polyp from being resected endoscopically. After receiving 2 units of red blood cells and 2 units offresh frozen plasma, the patient was transferred on the surgical ward due to the on going hemorrhage. Even though an $18 \mathrm{~cm}$ diameter reducible incisional hernia sac was present in the central part of the abdomen, we decided to use the laparoscopic approach for 2 reasons: first of all, for avoiding to create an even bigger abdominal defect, since the existing one couldn't be corrected during the same procedure and for eliminating the risk of intraoperative bleeding from a big incision, as it usually happens in an open approach.

\section{Surgical setting}

The patient was placed in the French position on the operating table, with the surgeon on the left, the camera operator between the patient's legs and the assistant on the right. The monitors were situated on the right-hand side of the patient and at her head. A nasogastric tube was placed after or tracheal intubation.

\section{Surgical technique}

The incisional hernia sac was opened through a $15 \mathrm{~mm}$ skin incision. The transverse colon and the greater omentum were loosely adherent to the skin. Carbon dioxide was insufflated at a pressure of $12 \mathrm{mmHg}$. The optical trocar was introduced through this incision and sutured to the skin (Figure 1). The camera was then advanced, going around the left of the viscera which were retracted in the hernia sac. The $5 \mathrm{~mm}$ working trocar was placed below the left costal margin on the midclavicular line. After dissecting some of the greater omental adhesions on the left side of the abdominal wall, another two $5 \mathrm{~mm}$ trocars were inserted: one below the xiphoid process and the other one was placed 2 fingerbreadths above the left iliac crest. The ultrasound aspect of the liver was also confirmed intraoperative by visualizing macronodular cirrhosis. Using the electro cautery, an incision was made on the anterior side of the pyloric antrum. The assistant packed the polyp and retracted it over the stomach, where its base was ligated with Vicryl 3-0 suture. Afterwards, the polyp was resected and the gastrostomy was closed with the same suture. A drain was externalized through the subcostal incision. The operating time was 110 minutes (Fig. 2). 


\section{Postoperative evolution}

The patient was able to ambulate on the first postoperative day. The nasogastric tube was removed the next day, when the patient also started oral alimentation and the intestinal transit resumed. She was discharged on the fifth postoperative day only because she suffered from motion sickness and lived 120 $\mathrm{km}$ away from the hospital. The histopathologic exam of the polyp showed no malignancy signs. The patient was afterwards followed up by the gastroenterology department.

\section{Case 2}

A 73 year-old female patient was admitted to the hospital through the emergency department for an intermittent (colicky) abdominal pain, fever $\left(38^{\circ} \mathrm{C}\right)$, shivers, nausea and vomiting. She also presented a 22 year-old massive irreducible umbilical hernia, associated with class 2 obesity $\left(38.4 \mathrm{~kg} / \mathrm{m}^{2}\right.$ body mass index) and a high-risk $3^{\text {rd }}$ grade arterial hypertension. The ultrasound examination showed an acute cholecystitis with up to $12 \mathrm{~mm}$ gallbladder stones and a wall thickness of $4 \mathrm{~mm}$. The physical exam of the patient reveals abdominal tenderness and discrete muscular spasm in the right hypochondrium, beside the $22 \mathrm{~cm}$ diameter umbilical hernia.

The blood work was within normal range. We decided to perform a laparoscopic cholecystectomy as a first surgery, since the bulky hernia wasn't tender on palpation. After one month, a second classic surgery would be performed in order to reduce the hernia and implant a polypropylene mesh. By separating these 2 procedures, the risk of contaminating the mesh during an associated cholecystectomy would be significantly reduced.

\section{Surgical preparation}

The patient was placed in a supine position on the operating table, with the surgical team in the standard position for a cholecystectomy with 4 ports.

\section{Surgical technique}

\section{Postoperative evolution}

The patient resumed her intestinal transit on the second postoperative day, when the peritoneal drain was also removed. She was discharged on postoperative day 3. A followup was scheduled a month after, when the umbilical hernia was operated.

\section{Results and Discussion}

Previous open abdominal surgery is no longer an absolute contraindication for laparoscopic surgery because, although it prolongs the operation time, postoperative outcomes are not different from patients without previous surgery $(5,6)$. The laparoscopic technique is feasible in patients with associated abdominal wall defects if the trocars are introduced safely (7) and in ergonomic position for the pathologies that are going to be addressed (8, 9 ). In the cases of reducible incisional hernia, a space through which the camera trocar can be introduced should be easily found by using Hasson`s technique (Figure 3). If needed, the incision can be extended.

In the cases of irreducible hernias, the best way to approach the abdomen is through an incision at the edge of the hernia sac and pass all the abdominal wall layers, using again Hasson`s technique (Figure 4). The rest of the ports are placed in a triangular fashion, avoiding shearing and having a manipulation angle of $45-75^{\circ}$ between the ports and the target organs $(8,10)$. 


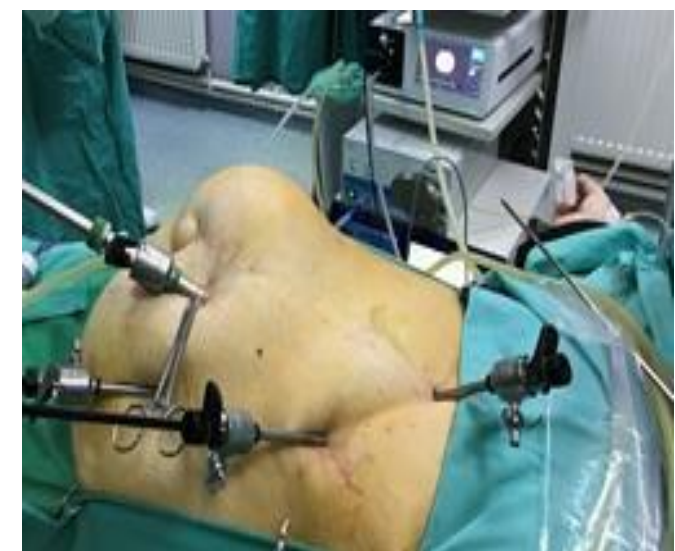

Figure.1 Trocars position in the laparoscopic resection of a gastric polyp through the incisional hernia sac.

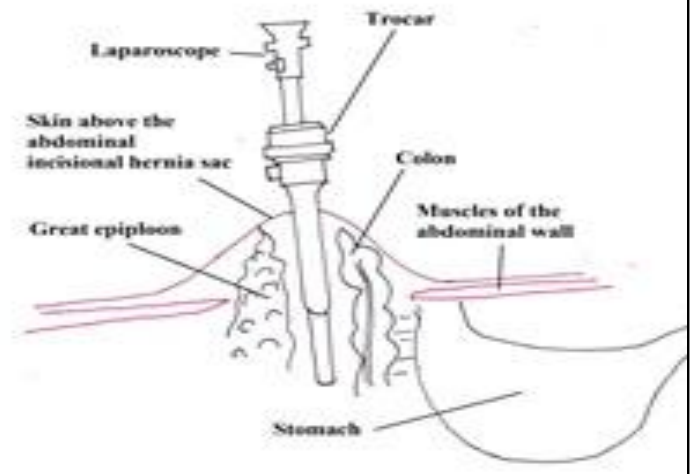

Figure.3 Laparoscope position in the incisional hernia sac.

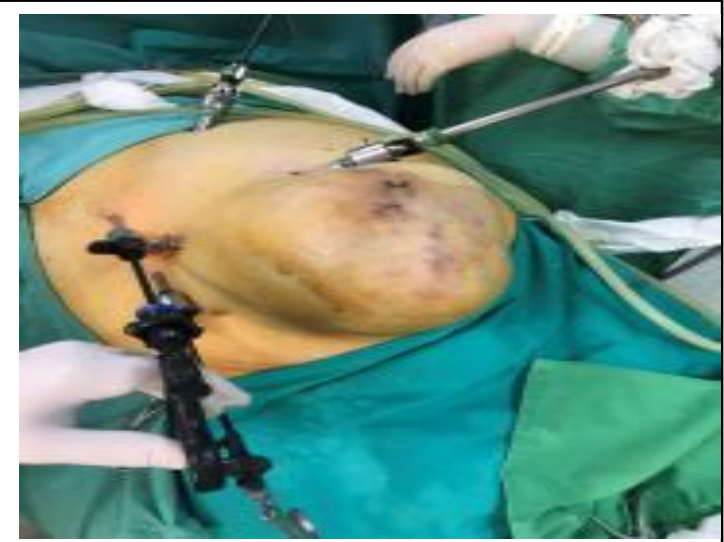

Figure.2 Trocars position in laparoscopic cholecystectomy in a patient with irreducible umbilical hernia

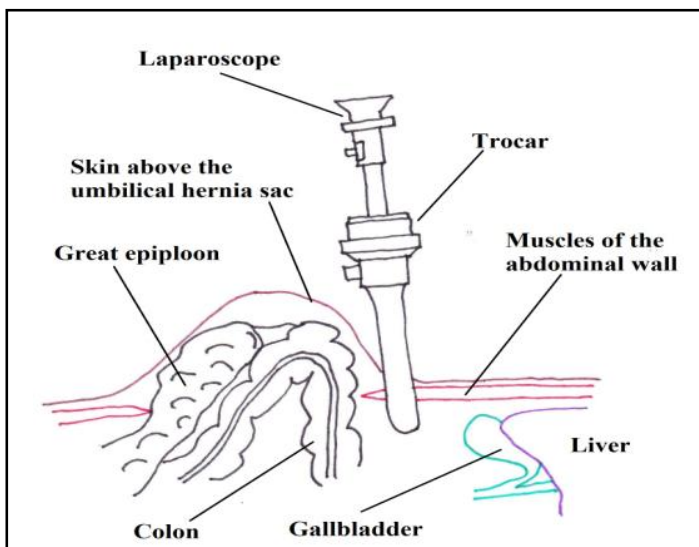

Figure.4 Laparoscope position at the edge of the umbilical hernia sac

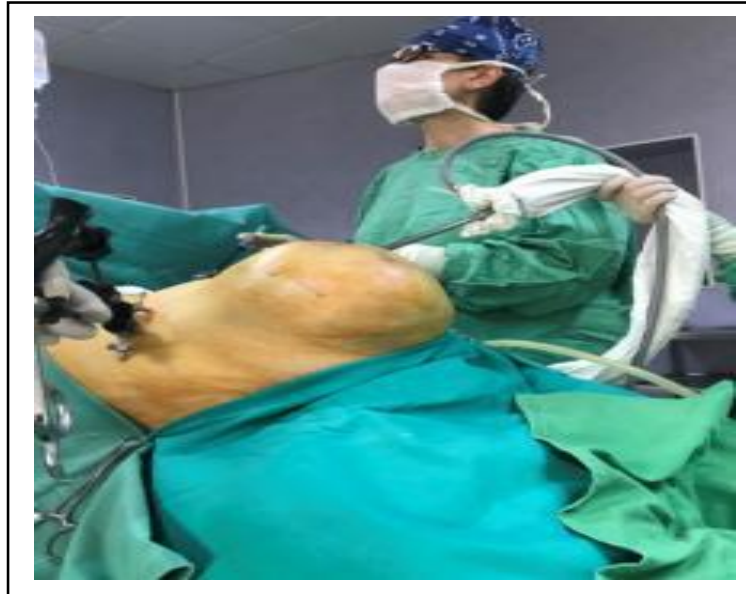

Figure.5 Laparoscopic cholecystectomy in a patient with giant umbilical hernia 
A multi-stage surgical treatment for patients with multiple associated surgical pathologies is recommended especially in emergency situations. In these cases, where the patients are in poor condition and one of the pathologies needs immediate surgical treatment, trying to address more than one condition at a time would unacceptably increase the risk of intra- and postoperative complications.

Working in an emergency hospital, we were often confronted with situations such as perforated ulcers, acute appendicitis, splenic or hepatic trauma we gave up laparoscopic approach due to anterior abdominal wall scars. The short and long term results were affected by multiple complications (wound infections, pneumonia, incisional hernia, etc.) which were difficult to treat as patients had also multiple associated diseases. With the gain of experience, we are now able to approach laparoscopic patients with intestinal occlusion due to adhesions, abdominal trauma or colon resection even if they had a previous open abdominal surgery.

The laparoscopic approach, in the hands of a trained team, can be an option for complex patients present with multiple associated diseases and high operative risk (11, 12). Although impressive (Figure 5), giant abdominal wall defects do not represent an absolute contraindication for the laparoscopic approach when a different condition needs immediate surgical intervention.

The surgical technique can be chosen based on the surgical team ability to perform laparoscopic surgery, the overall condition of the patients and the availability of the technical equipment. Giant abdominal wall defects and adhesions are not absolute contraindications for the laparoscopic treatment of other pathologies in need of emergency surgical intervention.

\section{References}

Coffin SJ, Wrenn SM, Callas PW, Abu-Jaish $\mathrm{W}$, Three decades later: investigating the rate of and risks for conversion from laparoscopic to open cholecystectomy, Surg Endosc, 2018 Feb; 32(2): 923-929.

Eker HH et al., Laparoscopic vs. open incisional hernia repair: a randomized clinical trial, JAMA Surg. 2013; 148(3): 259-263.

Geraci G, D’Orazio B, Rizzuto S, Cajozzo M, Modica G, Videolaparoscopic cholecystectomy in patients with previous abdominal surgery. Personal experience and literature review, Clin Ter, 2017; 168(6): e357-360.

Harrysson IJ, Cook J, Sirimanna P, Feldman LS, Darzi A, Aggarwal R, Systematic review of learning curves for minimally invasive abdominal surgery: a review of the methodology of data.

collection, depiction of outcomes, and statistical analysis, Ann Surg. 2014 Jul; 260(1): 37-45.

Hasson HM. A modified instrument and method for laparoscopy. Am J Obstet Gynecol 1971; 110: 886-7.

Lee SY, Kim CH, Kim YJ, Kim HR, Laparoscopic surgery for colorectal cancer patients who underwent previous abdominal surgery, Surg Endosc, 2016 Dec;30(12):5472-5480.

Levy B, Mohammad Mobasheri M, Principles of safe laparoscopic surgery, Surgery, Apr 2017; 35(4):216-219.

Nozaki I, Kubo Y, Kurita A, Ohta K, Aogi K, Tanada M, Takashima S, Laparoscopic colectomy for colorectal cancer patients with previous abdominal surgery, Hepatogastroenterology, 2008 May-Jun; 55(84): 943-6

Sarker SK1, Patel B, Simulation and surgical training, Int J Clin Pract. 2007 Dec; 61(12):2120-5. 
Supe AN, Kulkarni GV, Supe PA, Ergonomics in laparoscopic surgery, $\mathrm{J}$ Minim Access Surg. 2010 Apr-Jun; 6(2): 31-36

Târcoveanu E1, Moldovanu R, Bradea C, Dimofte G, Lupaşcu C, Georgescu S, Andronic D, Lotz JC, Vlad N, Vasilescu A, Laparoscopic surgical education - the experience of the first surgical unit Iaşi, Chirurgia (Bucur). 2011 Jan-Feb; 106(1):67-76

von Renteln D, Vassiliou MC, Rösch T, Rothstein RI, Triangulation: the holy grail of endoscopic surgery?, Surg Endosc. 2011 May; 25(5): 1355-7

\section{How to cite this article:}

Mihaela Leșe 2019. Laparoscopic approach through incisional or umbilical hernia sac. Int.J.Curr.Microbiol.App.Sci. 8(02): 3197-3202. doi: https://doi.org/10.20546/ijcmas.2019.802.373 